\title{
The Establishment of Intelligent Detection Method and Monitoring System for Underwater Target Based on Imaging Sonar
}

\author{
Pengfei Peng ${ }^{1, a}$, Qian $\mathrm{Yu}^{1}$, Qiyuan $\mathrm{Li}^{1}$ \\ ${ }^{1}$ School of Electronic Engineering, Naval University of Engineering, \\ Liberation Road NO.717, Wuhan 430033, Hubei, China
}

\begin{abstract}
For the practical requirement of underwater safety protection, the conception of target precautionary area is put forward combined with the technical characteristic of imaging sonar and the analysis of small underwater target imaging feature. And a detection method for underwater moving target based on image processing is build up, so that the intelligent detection and recognition of the underwater specific target is realized. Meanwhile, the intelligent detection and monitoring system of underwater target based on imaging sonar is designed and developed with the use of multi-level component-based architecture according to the practical application requirements. The system has obtained remarkable economic benefit in practical use and has good prospects for application.
\end{abstract}

\section{Introduction}

Underwater safety problem has always been the weak link in security field of all countries. Criminals take advantage of the characteristics of "asymmetric" war-fare to attack on offshore facilities by using small arms such as frogmen, frogman vehicles and underwater robots, which can cause a great loss of life and property. With the miniaturization of underwater weapon and equipment, it has become the key problem needed to be solved urgently of future underwater security to build the special underwater monitoring system for detecting small targets such as frogmen and underwater robots [1].

The existing imaging sonar equipment is used by the intelligent detection and monitoring system for underwater target to build the underwater target detection network based on the imaging sonar [2, 3]. Functions, such as the intelligent identification, monitoring and warning of underwater target, which provide effective means for real-time monitoring of underwater target of important ports, are realized by using intelligent technologies, such as image compression, dynamic target detection and composite locating and tracking.

Small underwater targets especially frogmen differ from the ones with metal shells. And the intensity of the target is affected by its own equipment, the angle, the environment, and other various factors. When frogmen acted underwater independent on the vehicle, only the high resolution imaging sonar could be used for detecting effectively. In addition, due to the need of implementing all-weather monitoring for underwater target in important area, it will be an onerous task for attendants to stare long at so many monitors. Especially when there are a lot of

\footnotetext{
${ }^{\mathrm{a}}$ Corresponding author: pengpengfei@126.com
}

warning points, this way not only increased the workload of operators on duty, and is difficult to guarantee the high safety and reliability, almost impossible to complete the comprehensive monitoring. Underwater monitoring system, there-fore, must have the functions, such as intelligence detection of underwater target, identification, location tracking, threat alert, so as to realize unattended operation.

In this paper, the conception of target precautionary area is put forward combined with the technical characteristic of imaging sonar and the analysis of small underwater target imaging feature. And a detection method for underwater moving target based on image processing is build up, so that the intelligent detection and recognition of the underwater specific target is realized. Meanwhile, the intelligent detection and monitoring system of underwater target based on imaging sonar is designed and developed with the use of multilevel component-based architecture according to the practical application requirements.

\section{The detection method of underwater moving target based on image processing}

\subsection{Basic ideas}

The basic idea of inter frame difference is used in the target motion detection algorithm [4], which can be divided into four steps: image preprocessing, image differencing, process after differencing, and target 
confirmation and extraction. The algorithm process is shown in Figure 1.

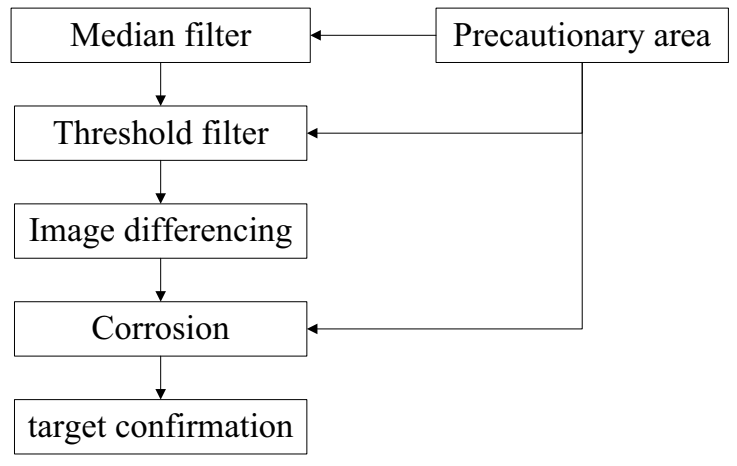

Figure 1. Algorithm process.

\subsection{Algorithm steps and implementation}

\subsubsection{Image preprocessing}

Median filter and threshold segmentation are mainly be used to remove the noise in the image [5-8]. Filter is mainly used to remove isolated noise point, and median filter can better keep the edge character of image, which is helpful to extract target and calculate related properties. Threshold segmentation is mainly for the background noise. The sonar is affected by the work environment, so background noise is stronger. The background noise can be removed by choosing appropriate threshold segmentation, thus the SNR (signal to noise ratio) of image is improved. For large-scale, continuous, highamplitude noise area, due to the low level concern of this part, the algorithm adopts the method of blocking to filter.

\subsubsection{Image differencing}

Through the method of inter frame difference the change of the part between two adjacent frames can be extracted, this section contains the potential moving target, the change of background, and the image jitter. For the sonar of fixed angle, the background is usually static. So the results of the inter frame difference method only include the potential moving target and the image jitter.

\subsubsection{Process after differencing}

Since the jitter of the image has the feature of isolation, the jitter of the image can be removed by means of etching, so that the results can mainly include the potential moving target. It should be noted that this post processing may also filter small targets.

\subsubsection{Target confirmation and extraction}

The last step of the algorithm is to confirm the target. According to the application environment of the project and the characteristics of the sonar image, in order to guarantee the reliability to extract the target, the algorithm is divided into three categories: large targets of strong echo intensity, small targets of strong echo intensity and large targets of weak echo intensity. In the process of the results, the targets are also divided into two categories: warning targets and confirmation tar-gets. For the images after differential processing, the average gray value, area, coordinates, and other attributes of the potential moving target region is calculated. When the attribute area and the average gray value of the potential moving target region are more than the threshold, the region is defined as the confirmation target. When the attribute area (large targets of weak echo intensity) or the average gray value of the potential moving target region (small targets of strong echo intensity) are more than the threshold, the region is defined as the warning target. Then according to the relevant information of the followup frames, the target is confirmed by the secondary confirmation method, and the target of the new confirmation is remarked as the marked target. The secondary confirmation method is that in the next frame, within a certain warning target region (the region range is generally set to the maximum movement distance of warning targets) the target whose attributes are similar to the warning target is extracted again and then the warning target is confirmed. The method of target confirmation and detection is shown in Figure 2.

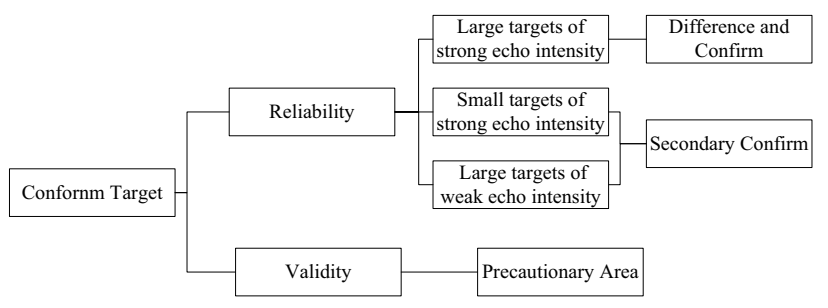

Figure 2. Target confirmation chart.

\subsection{The proposing and application of the precautionary area}

The key underwater targets of detection and monitoring are frogmen with the small-target characteristic. In order to guarantee the validity of the algorithm, the concept of the precautionary area is put forward based on the observation of the large number of image samples. Precautionary area includes edge precautionary area and target precautionary region. The edge precautionary area is such a region that when the target is near the sonar in a certain direction, the gray change of the edge of the sonar image is obvious. Therefore, the possible direction of target can be confirmed by comparing the change of target edge between the two frames. When the gray change of the edge of the sonar image is obvious, the area can be judged as the precautionary area. After the precautionary area is obtained, the algorithm takes the precautionary area as the initial area, and estimates next frame's possible moving region of the target (The region is obtained by the maximum movement speed and direction of the target, as shown in Figure 3). In the possible moving region of the target, the smaller median filter template and the lower segmentation threshold $\mathrm{s}$ used in the algorithm to improve the ability of the algorithm to extract small targets. The precautionary area 
of target is mainly for small targets, including confirmed small targets and potential small targets. The target precautionary area is based on such a reasoning: the next frame of true target will reappear near to the location of the target in the current frame, when a small target of strong echoes is extracted as a potential target, or a small target is confirmed, to which as the center the next frame's possible moving region of the target is estimated. And this region is considered as the precautionary area of the target. In the target's precautionary area, the small median filter template and the lower segmentation threshold are adopted similarly thus the ability of the algorithm to extract small target is improved.

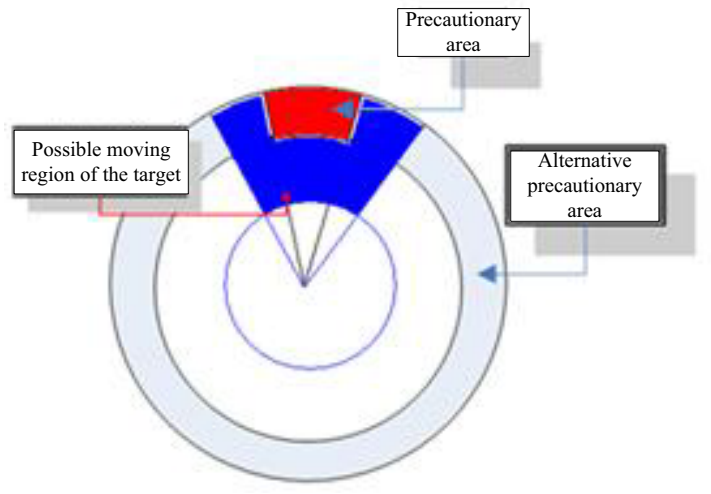

Figure 3. Diagram of edge precautionary area.

\section{The structure design and functions of intelligent detection and monitoring system for underwater targets}

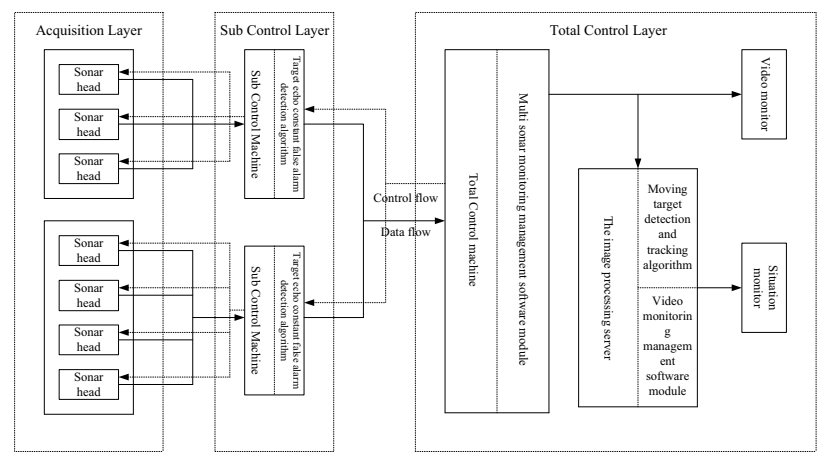

Figure 4. Overall framework of underwater target intelligent monitoring system.

As shown in Figure 4, the underwater target intelligent detection and monitoring system can be divided into three layers: acquisition layer, sub control layer, and total control layer. The acquisition layer is connected to the front imaging sonar to complete data acquisition work. One collection point can be responsible for multiple front imaging sonar. The existed Ethernet LAN (or small local area network) and the standard RS232/RS485 interface and IPX, TCP/IP transmission technology is used to complete data transmission. The image, the status signal is transmitted to the upper (the sub control layer and the total control layer), while the upper level sends out the control signal back to the front imaging sonar. Sub control layer: mainly accept the data of transport layer, display the original video of the first layer, and complete the information receiving of video recording sensor state, the sending of remote control instructions and other functions; Total control layer: mainly accept the data of video monitoring layer, image analysis and display the second layer, and complete the management and control of sub sites.

Based on the function of original standard video monitoring system, automatic detection and recognition module for mobile target is added in this system, including automatic alarm, alarm linkage video, alarm information display and query, target attribute recognition, target tracking $[9,10]$ and other functions.

\section{Conclusion}

In the actual application, dual frame difference image target detection method based on the precautionary area division has been successfully applied to the intelligent detection and monitoring system for underwater targets. The algorithm overcomes limitation of timeliness of the three frame method and the accumulated frame difference method in processing "empty" and "double image shadow" phenomenon, such the intelligent detection and monitoring for underwater target can be quickly and effectively solved. In addition, the existed small underwater target detection algorithms based on sonar imaging sequences are mostly based on the simple integration of image gray and size, and the detection algorithm based on active contour model can fuse multiple information, such as image regional features, the edge of the image, and the shape features of the target. This information fusion, especially the fusion of target shape information, can further improve the success rate of target detection. However, this algorithm has the disadvantages of large computation, and how to improve the operation efficiency and make it practically applied in system construction will be the main issue that needs to be studied next step.

\section{References}

1. A.M.Crawford, D.V.Crowe. Observations from Demonstrations of Several Commereial Diver Deteetion Sonar Systems[C]. Oceans2007, 2007.

2. T.Ruiz, Y.Petillot, David. L,. Bell J. Tracking objects in underwater multibeam sonar images[C]. IEE.

3. K.Steven, S.John. Improved active sonar detection using autoregressive prewhiteners[J]. J.Acoust.Soc.Amer.,1990,87(4):1603-1611.

4. V.Carmillet, P-O.Amblard, Jourdain G. Detection of Phase- or Frequency-Modulated signals in Reverberation Noise[J]. J. Acoust.Soc. Amer., 1999, 105(6): 3375-3389

5. G.Ming, A.A. Douglas. Using McDaniel's Model Represent Non-Rayleigh Reverberation[J]. IEEE Journal of Oceanic Engineering, 2001, 26(3): $348 \sim 357$.

6. K.Steven, S.John. Improved active sonar detection using autoregressive prewhiteners[J]. Journal of 
Acoustical Society of America, 1990. 87(4): 1603 -

1611.

7. V.Carmillet, G.Jourdain. Wideband sonar detection in reverberation using auto-regressive models[C]. in: Ocean MTS'96, Fort Lauderdale, 1996: 1435 1440.

8. B.Kalyan, A.Balasuriva. Sonar based automatic target detection scheme for underwater environments using CFAR techniques: a comparative study[C]. in: UT '04. 2004 International Symposium on, 2004: 33 37.

9. F.Jurie. Tracking objects with a recognition algorithm. Pattern Recognition Letters, 1998, 19(3-4): 331-340.

10. T.Drummond, R.Cipolla. Real-time visual tracking of complex structures. IEEE Trans Pattern Analysis and Machine Intelligence, 2002, 24(7): 932-946. 